\title{
Caractéristiques des bases de données sur les services médicaux au Canada
}

\author{
L. M. Lix, Ph. D. (1); R. Walker, M. Sc. (2); H. Quan, Ph. D. (2); R. Nesdole, M. Éd. (1); J. Yang, B. Sc. (3); G. Chen, Ph. D. (2); \\ pour l'équipe chargée de la surveillance et des résultats en matière d'hypertension du Groupe de travail PECH/CHEP
}

Cet article a fait l'objet d'une évaluation par les pairs.

\section{Résumé}

Introduction : Les bases de données sur les services médicaux (BDSM) constituent une ressource précieuse pour la recherche et la surveillance au Canada. Toutefois, étant donné que les provinces et les territoires entretiennent des bases distinctes, les éléments de données ne sont pas uniformisés. Dans cette étude, nous comparons les principales caractéristiques des BDSM.

Méthodologie : La source primaire a été une enquête auprès d'informateurs clés, ce qui a permis de recueillir des renseignements sur les années de disponibilité des données, les caractéristiques des patients et des fournisseurs de soins, les éléments inclus dans les bases de données et ceux exclus ainsi que le codage des diagnostics, des interventions et des lieux de prestation des services. On a également utilisé des données provenant de la Base de données nationale sur les médecins de l'Institut canadien d'information sur la santé (ICIS) pour examiner les modes de rémunération des médecins, ce qui peut influer sur l'exhaustivité des BDSM. Les données d'enquête ont été obtenues pour neuf provinces et deux territoires.

Résultats : La plupart des bases de données renfermaient des fichiers postérieurs à 1990 . Les diagnostics étaient fréquemment inscrits au moyen des codes de la CIM-9. Différents systèmes de codage ont été utilisés selon les provinces et territoires et selon les périodes; néanmoins, dans toutes les BDSM, les services aux malades hospitalisés ont été indiqués comme tels et la médecine familiale a été distinguée des autres spécialités. L’inscription des services rémunérés autrement qu'à l'acte présentait des différences, et les données de l'ICIS révélaient une proportion croissante, au fil du temps, des médecins non rémunérés à l'acte.

Conclusion : Il faut procéder à des recherches plus approfondies pour étudier les effets que pourraient avoir les différences entre les BDSM sur la comparabilité des résultats des études pancanadiennes.

Mots clés : Bases de données administratives sur la santé, services médicaux, programmes d'assurance-santé, Classification internationale des maladies

\section{Introduction}

Les données administratives sur la santé, recueillies dans le but de surveiller et de gérer les systèmes de santé, constituent une ressource précieuse pour la recherche et la surveillance au Canada. Les données proviennent de secteurs multiples, notamment des systèmes d'inscription à l'assurance-santé, des services aux malades hospitalisés, des services des urgences, des régimes de soins médicaux, des dossiers de statistiques de l'état civil et des systèmes de médicaments prescrits sur ordonnance. De plus en plus, on se sert de données administratives pour mener des études à l'échelle du Canada sur la santé de la population et sur l'utilisation des services de santé. Par exemple, le Système national de surveillance des maladies chroniques de l’Agence de la santé publique du Canada se fonde sur les diagnostics inscrits dans les dossiers hospitaliers et médicaux pour estimer la prévalence et l'incidence d'affections telles que le diabète et l'hypertension pour l'ensemble des provinces et des territoires du Canada ${ }^{1-3}$. On a également entrepris des études sur les maladies chroniques englobant plusieurs provinces et qui font appel aux données administratives sur les maladies rhumatismales, la maladie intestinale inflammatoire et les troubles mentaux ${ }^{4-6}$; d'autres recherches, notamment une étude sur l'hypertension ${ }^{7}$, sont en cours. Les données administratives sur la santé sont intéressantes pour la recherche et la surveillance car elles fournissent une solution de rechange économique à la collecte de données primaires, en plus d'englober des populations entières et de s'échelonner sur plusieurs années.

Malgré ses nombreux avantages, l'utilisation des données administratives sur la santé comporte des difficultés. Le Canada est doté d'un système de soins de santé universel, mais la prestation des services est de compétence provinciale et territoriale. La collecte et la gestion de la plupart des

Rattachement des auteurs :

1. School of Public Health, University of Saskatchewan, Saskatoon (Saskatchewan), Canada

2. Department of Community Health Sciences, University of Calgary, Calgary (Alberta), Canada

3. Western College of Veterinary Medicine, University of Saskatchewan, Saskatoon (Saskatchewan), Canada

Correspondance : Lisa Lix, School of Public Health, University of Saskatchewan, 107 Wiggins Road, Saskatoon (Saskatchewan) S7N 5E5; tél. : 306-966-1617; téléc. : 306-966-7920; courriel : lisa.lix@usask.ca 
données administratives se font à partir de systèmes d'information élaborés par les diverses provinces et les territoires, d'où un possible manque d'uniformisation et d'harmonisation dans les méthodes de collecte et de consignation des données. Font exception à cette règle les bases de données hospitalières nationales élaborées par l'Institut canadien d'information sur la santé (ICIS), notamment la Base de données sur les congés des patients et la Base de données sur la morbidité hospitalière, qui font appel à un formulaire commun de résumé analytique de dossier et à la même méthodologie d'évaluation de la qualité.

La qualité des données est un sujet de recherche pertinent dans le contexte actuel, alors que d'imposantes bases de données sont fréquemment utilisées pour la prise de décisions et l'élaboration de politiques ${ }^{8}$. Les chercheurs, les épidémiologistes et les décideurs intéressés à entreprendre des études pancanadiennes pourraient tirer parti des résultats des comparaisons entre les données administratives sur la santé venant de différentes provinces et différents territoires. Le fait de disposer d'information sur les caractéristiques de ces données peut faciliter la mise au point de méthodologies d'évaluation de la qualité et de protocoles de recherche permettant d'explorer l'effet potentiel des différences sur les conclusions des études. Les bases de données sur les services médicaux (BDSM), qui renferment les relevés d'honoraires ou les demandes de remboursement pour les consultations des médecins, revêtent une importance toute particulière pour la recherche et les activités de surveillance concernant les maladies chroniques. Elles constituent une source d'information sur les diagnostics posés dans le cadre des consultations externes et sur les interventions connexes, et elles renferment des renseignements concernant les consultations aussi bien des fournisseurs de soins primaires que des médecins spécialistes. Dans ce contexte, nous avons entrepris cette étude pour comparer les principales caractéristiques des BDSM des différentes provinces et des territoires du Canada. Une telle étude est essentielle si l'on veut disposer d'un point de référence pour les connaissances scientifiques. En outre, cette étude demeurera vraisemblablement pertinente pendant un certain temps du fait que bon nombre des caractéristiques des bases de données, notamment la première année de disponibilité des données pour les chercheurs, la version de la Classification internationale des maladies (CIM) utilisée, le nombre de champs de diagnostic, la spécificité des champs de diagnostic et la disponibilité des renseignements sur les services reçus à l'étranger, sont peu susceptibles de changer rapidement; elles sont de fait demeurées stables dans plusieurs provinces et territoires au cours des dernières années.

\section{Méthodologie}

La source primaire de données pour cette étude était un questionnaire d'enquête, envoyé par courriel aux informateurs clés de toutes les provinces et des territoires, et qui visait à recueillir de l'information sur certaines caractéristiques des BDSM. On a par ailleurs utilisé comme source secondaire de données les rapports publiés par l'ICIS, qui ont servi à réunir de l'information sur les modes de rémunération des médecins, un facteur qui peut influer sur l'exhaustivité des BDSM${ }^{8}$.

Les informateurs clés ont été principalement recrutés parmi les membres de l'équipe chargée de la surveillance et des résultats en matière d'hypertension (Hypertension Outcomes and Surveillance Team, ou HOST) $^{9}$, un sous-groupe du groupe de travail en charge de la recherche sur les résultats du Programme éducatif canadien sur l'hypertension. L'équipe HOST comprend environ 20 chercheurs, analystes et représentants gouvernementaux de la Colombie-Britannique, de l'Alberta, de la Saskatchewan, du Manitoba, de l'Ontario et du Québec, ainsi que de l'Agence de la santé publique du Canada et de Statistique Canada. Les membres de l'équipe HOST possèdent une expertise dans la recherche et la surveillance portant sur les services de santé ou sur la santé de la population et faisant appel à des données administratives. Dans le cas des provinces et territoires qui ne sont pas représentés au sein de l'équipe HOST, nous avons communiqué avec des collaborateurs de l'équipe HOST, c'est-à-dire des fonctionnaires des ministères provinciaux de la Santé chargés de faciliter l'accès aux données administratives et à la documentation à des fins de recherche.
On a communiqué avec une ou deux personnes de chaque province et territoire pour solliciter leur participation à l'étude.

Les chercheurs concernés ont bâti l'enquête sur la base des recherches antérieures sur les caractéristiques des données administratives sur la santé ${ }^{10}$. Les questions de l'enquête portaient sur les points suivants : années où des données sont disponibles pour les besoins des chercheurs, disponibilité, contenus des champs fournissant de l'information sur les patients et les fournisseurs de soins, codes de diagnostic et d'intervention, services fournis à l'extérieur de la province et services dispensés par des professionnels rémunérés autrement qu'à l'acte. Les barèmes d'honoraires des médecins ont également été une source d'information sur les modes de rémunération $^{11}$. Pour l'ensemble de l'enquête, on s'est servi de questions à réponse ouverte.

Le premier contact avec les informateurs clés a eu lieu en juin 2010. On a ensuite utilisé des questions de suivi et de la documentation en ligne au sujet des BDSM pour clarifier les réponses et élaborer des questions supplémentaires. En novembre 2010, on a envoyé aux informateurs clés un deuxième questionnaire dans le but d’obtenir des précisions sur les caractéristiques des BDSM.

Des données secondaires publiées par l'ICIS ont fourni de l'information supplémentaire sur les fournisseurs de soins payés selon un mode autre que la rémunération à l'acte (services cliniques rémunérés autrement) et sur les paiements effectués pour de tels services $^{12-14}$. Les données étaient extraites de rapports fondés sur la Base de données nationale sur les médecins, qui contient des données agrégées sur la rémunération des médecins provenant des régimes de soins médicaux des provinces et des territoires. Des rapports antérieurs contenaient de l'information sur la Base de données nationale sur les médecins et sur les méthodes de collecte de données ${ }^{12,15}$.

Le Comité d'éthique de la recherche de l'Université de Calgary a fourni l'approbation éthique requise pour le projet HOST, lequel comporte l'extraction et l'analyse de données administratives sur la santé provenant des provinces et 
des territoires, ainsi que la collecte et la publication de documents à l'appui des données administratives sur la santé (examen éthique $\mathrm{n}^{\circ}$ E188889). Pour l'analyse des données publiques provenant de l'ICIS, aucune approbation éthique n'était nécessaire.

\section{Résultats}

Les informateurs clés de toutes les provinces et des territoires ont répondu à l'enquête, à l'exception du NouveauBrunswick, qui n'a pas fourni de réponses, et des Territoires du NordOuest, qui n'ont fourni que des renseignements partiels. Par conséquent, le Nouveau-Brunswick et les Territoires du Nord-Ouest ont été exclus de l'analyse des données d'enquête.

\section{BDSM : années de disponibilité des données}

Selon les résultats de l'enquête, les BDSM du Canada renferment des données remontant jusqu'en 1970 (tableau 1); l'intervalle entre les premières années de disponibilité des données était de 24 ans. Les bases de données du Manitoba et de la Saskatchewan renferment les dossiers les plus anciens, datant du début des années 1970. Dans le cas de quatre provinces, les bases de données contiennent des dossiers remontant aux années 1980.
Cependant, le Québec et l'Alberta ont indiqué que certains des dossiers les plus anciens ne pouvaient être consultés facilement $\mathrm{du}$ fait qu'ils avaient été archivés dans un format différent de celui des dossiers récents. Pour les autres provinces et territoires, les bases de données renfermaient des dossiers datant des années 1990.

\section{Information sur les patients et les fournisseurs de soins}

L'enquête a également révélé que toutes les BDSM contiennent des identificateurs uniques pour les patients et les fournisseurs de soins (tableau 1). Avec la permission

TABLEAU 1

Principales caractéristiques des bases de données sur les services médicaux par province ou territoire : disponibilité des données, patients, fournisseurs de soins

\begin{tabular}{|c|c|c|c|c|c|}
\hline $\begin{array}{l}\text { Province/ } \\
\text { territoire }\end{array}$ & $\begin{array}{l}\text { Première année de } \\
\text { disponibilité des } \\
\text { données pour les } \\
\text { chercheurs }\end{array}$ & $\begin{array}{l}\text { Identificateurs } \\
\text { des patients }\end{array}$ & $\begin{array}{c}\text { Caractéristiques } \\
\text { personnelles des } \\
\text { patients }^{\mathrm{a}}\end{array}$ & $\begin{array}{l}\text { Identificateurs } \\
\text { des fournisseurs } \\
\text { de soins }\end{array}$ & Catégories et spécialités des fournisseurs de soins \\
\hline Alberta & $\begin{array}{l}\text { 1982; les données } \\
\text { antérieures à } 1994 \\
\text { sont présentées dans } \\
\text { un format différent }\end{array}$ & $\checkmark$ & $\checkmark$ & $\checkmark$ & $\begin{array}{l}\text { Plus de } 60 \text { catégories réparties en médecine familiale, spécialités } \\
\text { médicales, spécialités chirurgicales, autres. Spécialités définies } \\
\text { selon le régime de paiement. }\end{array}$ \\
\hline Saskatchewan & 1971 & $\checkmark$ & $\checkmark$ & $\checkmark$ & $\begin{array}{l}\text { Plus de } 70 \text { catégories réparties en médecine familiale, spécialités } \\
\text { médicales, spécialités chirurgicales, autres. Spécialités définies selon } \\
\text { la certification des médecins. }\end{array}$ \\
\hline Manitoba & 1970 & $\checkmark$ & $\checkmark$ & $\checkmark$ & $\begin{array}{l}\text { Plus de } 80 \text { catégories réparties en médecine familiale, spécialités } \\
\text { médicales, spécialités chirurgicales, autres. Champs définis selon } \\
\text { des regroupements de spécialités. }\end{array}$ \\
\hline $\begin{array}{l}\text { Île-du-Prince- } \\
\text { Édouard }\end{array}$ & $\begin{array}{l}\text { 1983; les données } \\
\text { antérieures à } 1996 \\
\text { sont présentées dans } \\
\text { un format différent }\end{array}$ & $\checkmark$ & $\checkmark$ & $\checkmark$ & $\begin{array}{l}\text { Plus de } 45 \text { descriptions de codes de spécialité réparties en médecine } \\
\text { familiale, spécialités médicales, spécialités chirurgicales, autres. } \\
\text { Spécialités définies selon les services dispensés. }\end{array}$ \\
\hline Nouvelle-Écosse & 1989 & $\checkmark$ & $\checkmark$ & $\checkmark$ & $\begin{array}{l}\text { Plus de } 50 \text { catégories réparties en médecine familiale, spécialités } \\
\text { médicales, spécialités chirurgicales, autres. Définies selon la } \\
\text { facturation et selon la principale spécialité. }\end{array}$ \\
\hline $\begin{array}{l}\text { Terre-Neuve-et- } \\
\text { Labrador }\end{array}$ & 1995 & $\checkmark$ & $\checkmark$ & $\checkmark$ & $\begin{array}{l}\text { Plus de } 80 \text { catégories réparties en médecine familiale, spécialités } \\
\text { médicales, spécialités chirurgicales, autres. Spécialités définies } \\
\text { selon la formation des médecins. }\end{array}$ \\
\hline
\end{tabular}

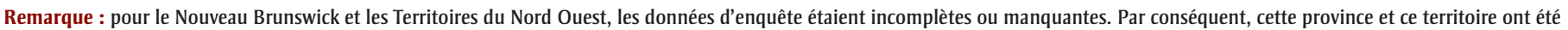
exclus de l'analyse.

${ }^{a}$ Comprennent la date de naissance ou l'âge, le sexe et le lieu de résidence (p. ex. code postal, région sanitaire ou comté). 
voulue, ces identificateurs peuvent servir à relier les bases de données à d'autres sources. Par exemple, on peut effectuer un couplage avec un registre de la population pour obtenir les dates de couverture des régimes d'assurance-santé des provinces, de l'information sur le lieu de résidence (p. ex. code postal ou zone géographique), la date de naissance ou l'âge ainsi que le sexe.

Dans toutes les bases de données sont également consignées les spécialités des fournisseurs de soins. Le nombre de catégories de spécialistes indiqué par les répondants allait d'environ 25 à plus de 80 . Toutefois, toutes les provinces et les territoires ont fait état, parmi les catégories mentionnées, de larges regroupements tels que la médecine familiale, les spécialités médicales et les spécialités chirurgicales. Parallèlement, on trouvait un mélange hétérogène de spécialités dans la catégorie « autre ». Selon la province ou le territoire, cette catégorie pouvait englober les praticiens des soins infirmiers, les sages-femmes, les pharmaciens et d'autres professions connexes de la santé. La plupart des répondants ont déclaré que la spécialité des fournisseurs de soins était attribuée par le régime de soins médicaux de chaque province ou territoire au moment où le relevé de service était soumis pour paiement.

\section{Information concernant les diagnostics et les interventions}

Le tableau 2 fournit de l'information sur les codes de diagnostic et d'intervention tels qu'ils ont été décrits par les répondants à

TABLEAU 2

Principales caractéristiques des bases de données sur les services médicaux au Canada : diagnostics et interventions

\begin{tabular}{|c|c|c|c|c|c|}
\hline $\begin{array}{l}\text { Province/ } \\
\text { territoire }\end{array}$ & $\begin{array}{l}\text { Version } \\
\text { de la CIM }\end{array}$ & $\begin{array}{l}\text { Nombre de champs } \\
\text { de diagnostic }\end{array}$ & $\begin{array}{l}\text { Spécificité des codes } \\
\text { de diagnostic } \\
\text { (nombre de chiffres) }\end{array}$ & $\begin{array}{l}\text { Source de } \\
\text { l'information } \\
\text { concernant } \\
\text { l'intervention }\end{array}$ & $\begin{array}{l}\text { Autre information sur les champs } \\
\text { de diagnostic/d'intervention }\end{array}$ \\
\hline $\begin{array}{l}\text { Colombie- } \\
\text { Britannique }\end{array}$ & CIM 9 & $\begin{array}{l}\text { La plupart des relevés } \\
\text { renferment un code, } \\
\text { mais certains médecins de } \\
\text { soins primaires inscrivent } \\
\text { jusqu'à } 3 \text { codes. }\end{array}$ & $\begin{array}{l}\text { Jusqu'à } 5 \text {, } \\
\text { mais on en trouve } \\
\text { le plus souvent } 3 \text {. }\end{array}$ & $\begin{array}{c}\text { Codes } \\
\text { d'honoraires }\end{array}$ & $\begin{array}{c}\text { Dans certains relevés, on trouve également les } \\
\text { codes de la CIM } 9 \text { MC. Certains codes de } \\
\text { diagnostic sont propres au régime d'assurance } \\
\text { santé de la province. }\end{array}$ \\
\hline Alberta & CIM 9 MC & 3 (1 avant 1994) & $\begin{array}{l}\text { Jusqu'à } 5 \text {, mais le } \\
5^{\text {e }} \text { chiffre n'est pas } \\
\text { bien inscrit; avant } \\
\text { 1994, on utilisait des } \\
\text { codes à } 3 \text { chiffres. }\end{array}$ & $\begin{array}{l}\text { Classification } \\
\text { canadienne des } \\
\text { interventions }\end{array}$ & \\
\hline Saskatchewan & $\begin{array}{l}\text { CIM } 8 \text { jusqu'en } \\
\text { 1978, puis CIM } 9\end{array}$ & 1 & 3 & $\begin{array}{l}\text { Codes } \\
\text { d'honoraires }\end{array}$ & $\begin{array}{l}\text { On trouve également certains codes de } \\
\text { diagnostic attribués par la province; certains } \\
\text { codes de la CIM } 9 \text { (notamment tous les codes } \\
\text { E et certains autres codes) ne sont pas utilisés. }\end{array}$ \\
\hline Manitoba & $\begin{array}{l}\text { CIM } 8 \text { jusqu'en } \\
\text { 1979, puis } \\
\text { CIM } 9 \text { MC }\end{array}$ & 1 & 3 & $\begin{array}{l}\text { CIM } 9 \text { MC et codes } \\
\text { d'honoraires }\end{array}$ & \\
\hline Ontario & $\begin{array}{l}\text { Mélange de CIM } \\
8 \text { et de CIM } 9\end{array}$ & 1 & $\begin{array}{l}\text { 3; un suffixe à } 1 \\
\text { chiffre est ajouté pour } \\
\text { la physiothérapie } \\
\text { et la chiropractie }\end{array}$ & Codes d'honoraires & $\begin{array}{l}\text { La base de données ne contient pas tous les } \\
\text { codes de diagnostic de la CIM } 8 \text { et de la CIM } 9 .\end{array}$ \\
\hline Québec & CIM 9 & 1 & 4 & $\begin{array}{l}\text { Codes propres } \\
\text { à la province }\end{array}$ & \\
\hline $\begin{array}{l}\text { Île-du-Prince- } \\
\text { Édouard }\end{array}$ & CIM 9 & 1 & $\begin{array}{l}\text { 3, à l'exception des } \\
\text { codes E et des codes V, } \\
\text { qui en ont } 5 .\end{array}$ & $\begin{array}{l}\text { CIM } 9 \text { et codes } \\
\text { d'honoraires }\end{array}$ & \\
\hline $\begin{array}{l}\text { Nouvelle- } \\
\text { Écosse }\end{array}$ & CIM 9 & 3 (1 avant 1997) & $\begin{array}{l}\text { Jusqu'à } 5 \text {; les codes à } \\
3 \text { chiffres, qui sont les } \\
\text { plus courants, étaient } \\
\text { utilisés avant } 1997 .\end{array}$ & $\begin{array}{l}\text { Version modifiée } \\
\text { de la Classification } \\
\text { canadienne des } \\
\text { interventions. Un seul } \\
\text { code est inscrit. }\end{array}$ & $\begin{array}{l}\text { Certains codes E sont inscrits dans un champ } \\
\text { de « diagnostic du traumatisme », mais on } \\
\text { ne sait pas si ces codes sont systématiquement } \\
\text { inscrits ni s'ils le sont au complet. }\end{array}$ \\
\hline $\begin{array}{l}\text { Terre-Neuve-et- } \\
\text { Labrador }\end{array}$ & CIM 9 & 1 & 3 & $\begin{array}{l}\text { Codes } \\
\text { d'honoraires }\end{array}$ & \\
\hline Yukon & CIM 9 + texte & $\begin{array}{l}2 \text { avant } 2006 \text {, } \\
\text { aucune limite depuis }\end{array}$ & $\begin{array}{l}\text { Jusqu'à } 5 \text {, mais la } \\
\text { plupart des relevés } \\
\text { n'en contiennent } \\
\text { que } 4\end{array}$ & $\begin{array}{l}\text { Codes } \\
\text { d'honoraires }\end{array}$ & \\
\hline Nunavut & CIM 9 MC & 11 & 5 & Aucun & $\begin{array}{l}\text { Les bases de données permettent l'inscription } \\
\text { des codes d'intervention, mais cette } \\
\text { information n'est pas saisie à l'heure actuelle. }\end{array}$ \\
\hline
\end{tabular}

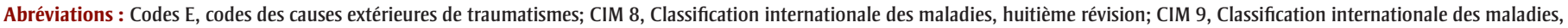
neuvième révision; CIM 9 MC, Classification internationale des maladies, neuvième révision, modification clinique; codes V, codes supplémentaires.

Remarque : pour le Nouveau Brunswick et les Territoires du Nord Ouest, les données d'enquête étaient incomplètes ou manquantes. Par conséquent, cette province et ce territoire ont été exclus de l'analyse. 
l'enquête. Toutes les provinces et les territoires ont utilisé les codes de la CIM pour consigner la majorité des diagnostics. En Saskatchewan et au Manitoba, dans les années 1970, on s'est d'abord servi des codes de diagnostic de la CIM-8* ${ }^{*}$ Le Manitoba, l'Alberta et le Nunavut ont utilisé les codes de la CIM-9-MC ${ }^{\dagger}$. Au Yukon, les diagnostics ont été consignés au moyen des codes de la CIM-9 ainsi que de texte de forme libre. L'Ontario s'est servie aussi bien des codes de la CIM-8 que des codes de la CIM-9, aucun des systèmes n'ayant cependant été utilisé dans son intégralité. En Saskatchewan, les codes de la CIM-9 n’ont pas tous été employés pour consigner les diagnostics.

Les répondants de toutes les provinces sauf trois ont affirmé qu'un seul diagnostic est consigné pour chaque demande de remboursement dans leur BDSM. En Colombie-Britannique, on peut trouver jusqu'à trois champs de diagnostic dans certaines des demandes. La base de données de la Nouvelle-Écosse contient, pour certaines années, trois codes de diagnostic différents. La base de données de l'Alberta renferme aussi trois champs de diagnostic, bien que les répondants aient souligné que le deuxième et le troisième champs n'étaient pas remplis de façon uniforme dans tous les fichiers. Les bases de données du Yukon et du Nunavut contiennent des champs de diagnostic multiples.

Les codes de diagnostic ont été saisis avec un degré de précision variable, les codes à trois chiffres étant les plus communs. Quoique l'information concernant les interventions ait été fournie le plus souvent au moyen des codes d'honoraires, d'autres systèmes de codage des interventions ont été utilisés, notamment la Classification canadienne des interventions en NouvelleÉcosse et en Alberta.

\section{Lieu de prestation des services}

On a constaté, d'après les réponses obtenues, que les BDSM ne contiennent pas toutes un ou des champs indiquant le lieu de prestation des services (voir le tableau 3); il faut parfois se reporter au code d'honoraires et au lieu de facturation pour connaitre le lieu de prestation des services. Toutefois, dans toutes les provinces et les territoires, il est possible de distinguer les services aux malades hospitalisés des autres types de services.

\section{Éléments inclus dans les BDSM et éléments exclus}

Le tableau 3 fournit de l'information, tirée de l'enquête, sur les relevés venant de fournisseurs de soins à l'extérieur de la province. Les répondants de quatre provinces (Terre-Neuve-et-Labrador, Nouvelle-Écosse, Manitoba et ColombieBritannique) ont signalé que ces fichiers étaient versés dans une base de données distincte. En ce qui concerne les autres provinces et territoires, les répondants ont indiqué qu'un code ou un champ particulier, à l'intérieur de la BDSM, pouvait être utilisé pour désigner les relevés venant de l'extérieur de la province.

Le dernier point de l'enquête portait sur la consignation des relevés venant des médecins rémunérés à l'acte et ceux des médecins non rémunérés à l'acte. À l'exception de Terre-Neuve-et-Labrador et du Québec, les répondants ont indiqué que les BDSM renfermaient des relevés pour les services provenant de médecins non rémunérés à l'acte. Toutefois, ces répondants ont également fait remarquer qu'il n'était pas toujours possible de déterminer si tous les relevés venant de médecins non rémunérés à l'acte étaient inclus. Ce problème peut être attribuable aux changements survenus dans les régimes de soins médicaux au fil du temps et/ou à un manque de documentation sur les autres régimes de rémunération. Dans six provinces ou territoires, les répondants ont affirmé que les BDSM renfermaient un champ permettant de distinguer les relevés soumis par des médecins rémunérés à l'acte des relevés soumis par des médecins rémunérés autrement. Dans trois provinces, il faut se reporter à un registre des fournisseurs de soins pour différencier ces deux types de relevés.

\section{Données de l'ICIS sur les modes de rémunération des médecins}

On s'est servi des données secondaires de l'ICIS pour examiner les modes de rémunération des médecins utilisés par les provinces et les territoires, ce qui pourrait influer sur l'exhaustivité des BDSM. Dans les territoires, ces données n'étaient pas toujours disponibles, et elles n'ont donc pas été prises en compte. Dans la figure 1, le pourcentage de médecins équivalents temps plein payés selon un mode autre que la rémunération à l'acte (services cliniques rémunérés autrement) est indiqué pour les années financières 1999-2000 et 2005-2006; cette dernière année est la plus récente qu'on puisse obtenir de l'ICIS. Ce pourcentage a connu une hausse dans toutes les provinces, à l'exception de la Colombie-Britannique et de Terre-Neuve-et-Labrador. La plus importante hausse absolue concerne le Nouveau-Brunswick (17,3 \%) et l'Île-du-Prince-Édouard (15,0 \%). Après regroupement des données pour les quatre provinces de l'Atlantique, le pourcentage des médecins équivalents temps plein rémunérés selon d'autres modes de paiement des services cliniques est passé de 20,3 \% à 25,6 \%. On a également observé une importante augmentation absolue en Saskatchewan (6,5 \%). La figure 2 fournit des données sur les autres modes de paiement des services cliniques aux médecins pour les années financières 1999-2000 et 2008-2009. Dans l'ensemble, la proportion occupée par ce type de paiements dans les quatre provinces de l'Atlantique est passée de 23,8 \% à 39,8\% . D'importantes hausses entre les deux années à l'étude ont également été constatées en Ontario et en Saskatchewan.

\section{Analyse}

Nous n’avons repéré qu'une seule autre étude publiée décrivant de façon systématique les caractéristiques des BDSM au Canada, étude portant sur la base de données de la Saskatchewan ${ }^{16}$. Toutefois, des études antérieures visant à comparer les bases de données administratives sur les médicaments d'ordonnance de plusieurs

\footnotetext{
* Classification internationale des maladies, huitième révision.

+ Classification internationale des maladies, neuvième révision, modification clinique.

₹ Classification internationale des maladies, neuvième révision.
} 
TABLEAU 3

Principales caractéristiques des bases de données sur les services médicaux au Canada : lieu de prestation des services et exhaustivité

\begin{tabular}{|c|c|c|c|c|}
\hline $\begin{array}{l}\text { Province/ } \\
\text { territoire }\end{array}$ & Catégories pour le lieu de prestation des services & $\begin{array}{l}\text { Services reçus à } \\
\text { l'extérieur de la } \\
\text { province }\end{array}$ & $\begin{array}{l}\text { Relevés soumis selon le } \\
\text { système de facturation } \\
\text { fictive }\end{array}$ & $\begin{array}{l}\text { Indicateur de } \\
\text { facturation fictive }\end{array}$ \\
\hline $\begin{array}{l}\text { Colombie- } \\
\text { Britannique }\end{array}$ & $\begin{array}{l}\text { Les lieux de prestation dans la province et à l'extérieur du pays } \\
\text { sont indiqués. Les lieux des services, y compris les hôpitaux et les } \\
\text { cliniques, sont disponibles à partir de } 1990 \text {. }\end{array}$ & $\begin{array}{l}\text { Inscrits dans une base } \\
\text { de données distincte }\end{array}$ & $\begin{array}{l}\text { Oui, mais on ne sait pas } \\
\text { si les renseignements } \\
\text { sont complets. }\end{array}$ & $\begin{array}{l}\text { Oui. À partir de 1999, les } \\
\text { factures fictives peuvent } \\
\text { être signalées au moyen } \\
\text { d'un indicateur. }\end{array}$ \\
\hline Alberta & $\begin{array}{l}\text { Les lieux de prestation des services sont repérables en fonction } \\
\text { de l'adresse. }\end{array}$ & $\begin{array}{l}\text { Indiqués dans un champ } \\
\text { de la base de données }\end{array}$ & $\begin{array}{l}\text { Oui, mais on ne sait pas } \\
\text { si les renseignements } \\
\text { sont complets. }\end{array}$ & Oui \\
\hline Saskatchewan & $\begin{array}{l}\text { Pour les services en cabinet, services aux malades hospitalisés, services } \\
\text { de consultation externe, services à domicile et autres, le lieu de } \\
\text { prestation n'est pas indiqué; médecins en salle d'urgence. Les codes } \\
\text { d'honoraires sont également utilisés pour désignner les services fournis } \\
\text { à l'hôpital. Le lieu de prestation des services est devenu un champ } \\
\text { obligatoire dans les années 1980, mais la date exacte est inconnue. Ce } \\
\text { champ n'est pas validé. }\end{array}$ & $\begin{array}{l}\text { Indiqués par un champ } \\
\text { dans la base de données }\end{array}$ & $\begin{array}{l}\text { Oui, mais on ne sait pas } \\
\text { si les renseignements } \\
\text { sont complets. }\end{array}$ & Oui \\
\hline Manitoba & $\begin{array}{l}\text { Les catégories de lieux de prestation dans les hôpitaux sont indiquées } \\
\text { dans un champ. Les services fournis dans d'autres lieux, y compris } \\
\text { les soins à domicile et les foyers de soins infirmiers, sont indiqués en } \\
\text { fonction des codes d'honoraires. Les services fournis en clinique sont } \\
\text { indiqués d'après le lieu de facturation. }\end{array}$ & $\begin{array}{l}\text { Indiqués dans une base } \\
\text { de données distincte. } \\
\text { Disponibles depuis } \\
\text { 1993-1994 }\end{array}$ & $\begin{array}{l}\text { Oui, mais on ne sait pas } \\
\text { si les renseignements } \\
\text { sont complets }\end{array}$ & $\begin{array}{l}\text { Non. Les factures fictives } \\
\text { peuvent être signalées au } \\
\text { moyen des renseignements } \\
\text { contenus dans le registre } \\
\text { des fournisseurs de soins. }\end{array}$ \\
\hline Ontario & $\begin{array}{l}\text { Dans les données actuelles, il n'y a pas de champ pour le lieu de } \\
\text { prestation des services. Les lieux sont indiqués à partir des codes } \\
\text { d'honoraires et/ou des numéros d'identification des établissements. } \\
\text { Un numéro d'identification maître pour les hôpitaux est inscrit pour } \\
\text { les services fournis à l'hôpital. }\end{array}$ & $\begin{array}{l}\text { Désignés par un code } \\
\text { numérique dans la base } \\
\text { de données ou par un } \\
\text { code d'honoraires ou le } \\
\text { numéro d'identification } \\
\text { du médecin }\end{array}$ & $\begin{array}{l}\text { Oui, mais on ne sait pas } \\
\text { si les renseignements } \\
\text { sont complets. }\end{array}$ & Oui \\
\hline Québec & $\begin{array}{l}\text { Deux lieux principaux : cabinets privés (p. ex. cabinet/clinique) et } \\
\text { établissements (p. ex. hôpital). Pour les établissements, on compte } \\
\text { plus de } 40 \text { catégories de lieux de prestation, dont établissements } \\
\text { privés, hôpitaux, services de laboratoire, diagnostic et radiologie, } \\
\text { cliniques de physiothérapie, centres d'accueil pour enfants et } \\
\text { adolescents, agences fédérales, universités, cliniques privées, } \\
\text { laboratoires privés d'orthotique prosthétique, centres de détention. }\end{array}$ & $\begin{array}{l}\text { Indiqués par un code } \\
\text { numérique dans la base } \\
\text { de données }\end{array}$ & Non & Non \\
\hline $\begin{array}{l}\text { Île-du-Prince- } \\
\text { Édouard }\end{array}$ & $\begin{array}{l}\text { On utilise actuellement } 25 \text { codes : cabinets, consultations à } \\
\text { domicile, services aux malades hospitalisés, services de consultation } \\
\text { externe, autres cabinets, chirurgies d'un jour, cliniques spécialisées, } \\
\text { établissements de soins communautaires, autres sites, cliniques de } \\
\text { l'UPEI, centres de désintoxication, cliniques « premier client », } \\
\text { radiologie pour malades hospitalisés, cliniques de nuit, radiologie } \\
\text { pour malades externes, spécialiste invité au Prince County Hospital, } \\
\text { spécialiste invité au Queen Elizabeth Hospital, cabinets ouverts le } \\
\text { samedi et le dimanche, radiologie, fournisseurs de soins dans tout } \\
\text { type d'établissement, urgence avec radiologie, cliniques sans rendez } \\
\text { vous, cabinets dentaires publics, cabinets dentaires privés, hygiénistes } \\
\text { dentaires publics. }\end{array}$ & $\begin{array}{l}\text { Indiqués par un code } \\
\text { numérique dans la base } \\
\text { de données }\end{array}$ & $\begin{array}{l}\text { Oui, mais on ne sait pas } \\
\text { si les renseignements sont } \\
\text { complets. Le } 1^{\text {er }} \text { juillet } \\
2008 \text {, on a instauré le } \\
\text { Clinical Work Incentive } \\
\text { (une mesure incitative en } \\
\text { faveur de la facturation } \\
\text { fictive); à partir de cette } \\
\text { date, les renseignements } \\
\text { sur la facturation fictive } \\
\text { sont plus complets. }\end{array}$ & $\begin{array}{l}\text { Non. Les factures fictives } \\
\text { peuvent être signalées au } \\
\text { moyen des relevés des } \\
\text { médecins ou du numéro } \\
\text { de spécialité. }\end{array}$ \\
\hline $\begin{array}{l}\text { Nouvelle- } \\
\text { Écosse }\end{array}$ & $\begin{array}{l}\text { Cabinets, centres correctionnels, soins à domicile dispensés par les } \\
\text { hôpitaux, soins à domicile, services d'hospitalisation, foyers de soins } \\
\text { infirmiers. Les lieux de prestation dans les hôpitaux comprennent : } \\
\text { désintoxication, salle des urgénces, soins intensifs, services aux } \\
\text { malades hospitalisés, unité néonatale, services de consultation externe. }\end{array}$ & $\begin{array}{l}\text { Inscrits dans une base } \\
\text { de données distincte }\end{array}$ & $\begin{array}{l}\text { Oui, mais on ne sait pas } \\
\text { si les renseignements } \\
\text { sont complets. }\end{array}$ & Oui, à partir de 1997 \\
\hline $\begin{array}{l}\text { Terre-Neuve- } \\
\text { et-Labrador }\end{array}$ & $\begin{array}{l}\text { Soins à domicile, cabinets, services aux malades hospitalisés, services } \\
\text { de consultation externe, salles des urgences. }\end{array}$ & $\begin{array}{l}\text { Inscrits dans une base } \\
\text { de données distincte }\end{array}$ & Non & Non \\
\hline Yukon & $\begin{array}{l}\text { Cabinets/cabinets de praticiens, soins à domicile, services aux malades } \\
\text { hospitalisés, services de consultation externe, laboratoires, cliniques } \\
\text { de spécialités chirurgicales, établissements de soins communautaires, } \\
\text { autres, cliniques à l'extérieur de la ville, foyers de soins infirmiers, } \\
\text { injection, anesthésie, services d'assistants chirurơicaux, admissions, } \\
\text { maternité, centres de détention. }\end{array}$ & $\begin{array}{l}\text { Inscrits dans un champ } \\
\text { de la base de données }\end{array}$ & $\begin{array}{l}\text { Oui, mais on ne sait pas } \\
\text { si les renseignements } \\
\text { sont complets. }\end{array}$ & Oui \\
\hline Nunavut & Aucun champ pour le lieu de prestation des services & $\begin{array}{l}\text { Désignés par un code } \\
\text { numérique dans la base } \\
\text { de données }\end{array}$ & Non & Non \\
\hline
\end{tabular}

Abréviation : UPEI, University of Prince Edward Island.

Remarque : Pour le Nouveau Brunswick et les Territoires du Nord Ouest, les données d'enquête étaient incomplètes ou manquantes. Par conséquent, cette province et ce territoire ont été exclus de l'analyse. 
FIGURE 1

Médecins équivalents temps plein payés selon un mode autre que la rémunération à l'acte (services cliniques rémunérés autrement), par province, 1999-2000 et 2005-2006

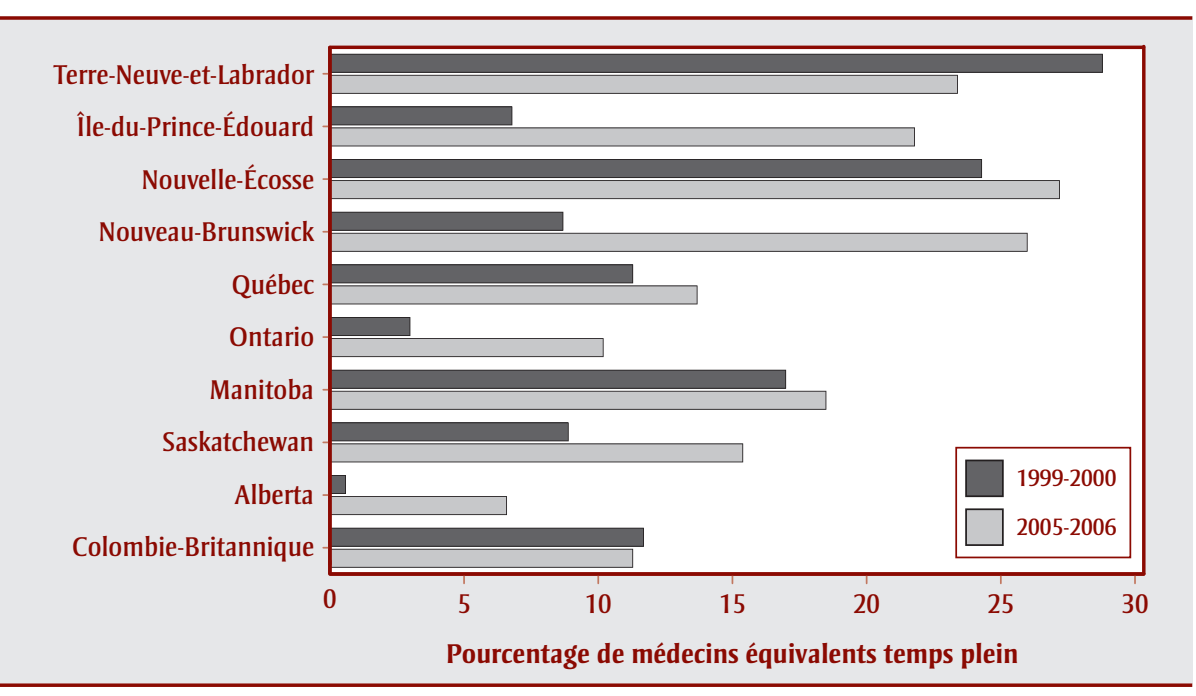

Source : Institut canadien d'information sur la santé. ${ }^{13,14}$

provinces canadiennes ont révélé des différences dans les caractéristiques des patients, des fournisseurs de soins et des médicaments ${ }^{10}$. Ces constatations sont conformes à celles de cette étude, qui ont mis en lumière l'hétérogénéité de bon nombre des caractéristiques des BDSM au Canada, notamment les années où des données sont disponibles, la classification des spécialités des fournisseurs de soins, les éléments inclus dans les bases de données et les éléments exclus, et le codage des diagnostics, des interventions et des lieux de prestation des services.

Les BDSM sont une source extraordinaire d'information dont peuvent tirer profit les activités de recherche et de surveillance portant sur la santé de la population et les services de santé au Canada. Le Système national de surveillance des maladies chroniques fait appel aux bases de données administratives sur la santé pour fournir des données longitudinales comparables sur la prévalence et l'incidence des maladies chroniques, et ainsi renforcer les mesures de surveillance mises en place par les provinces et les territoires. L'Initiative sur les données longitudinales administratives et sur la santé de Statistique Canada relie les bases de données administratives sur la santé avec les données des enquêtes sur la santé de la population, notamment l'Enquête nationale sur la santé de la population et l'Enquête sur la santé dans en partie, à des artefacts de mesures découlant des changements survenus dans les systèmes de codage (CIM), plutôt que refléter de véritables changements dans la distribution de la maladie au sein de la population ${ }^{18}$. La saisie incomplète des services dispensés par les médecins non rémunérés à l'acte peut entraîner un biais dans les estimations des tendances temporelles et des différences entre régions sanitaires $^{19}$. Le nombre d'années au cours desquelles des données sont disponibles peut influer sur les résultats des études portant sur la durée d'exposition à une maladie donnée et sur le délai avant l'apparition de la maladie ${ }^{20}$. Les champs de diagnostic sous forme de texte, tels ceux relevés dans la BDSM du Yukon, peuvent exiger le recours à des techniques d'exploration des données pour l'attribution des codes de diagnostic ${ }^{21}$, ce qui implique une méthodologie différente de celle utilisée dans d'autres provinces ou territoires pour confirmer les cas de maladie.

faciliter la recherche pancanadienne sur les déterminants de la santé et les résultats en matière de santé, et sur la relation entre ces deux éléments ${ }^{17}$.

Cependant, les différences relevées dans les BDSM à l'échelle du Canada sont un point important à considérer si l'on veut pouvoir comparer les conclusions des études de recherche et de surveillance. Les changements constatés au fil du temps dans les estimations de la prévalence des maladies peuvent être attribuables,

FIGURE 2

Certaines études pourraient ne pas être réalisables dans toutes les provinces et tous les territoires du Canada en raison des différences observées dans les systèmes de codage des données. Par exemple, certaines formes d'arthrite ne peuvent pas être consignées à l'aide des trois premiers chiffres de la CIM-922. Les BDSM de l'Ontario et de la Saskatchewan ne contiennent pas tous les codes, qu'il s'agisse de la CIM-8 ou de la CIM-9, un

Paiements selon un mode autre que la rémunération à l'acte (services cliniques rémunérés autrement) versés aux médecins, par province, 1999-2000 et 2008-2009

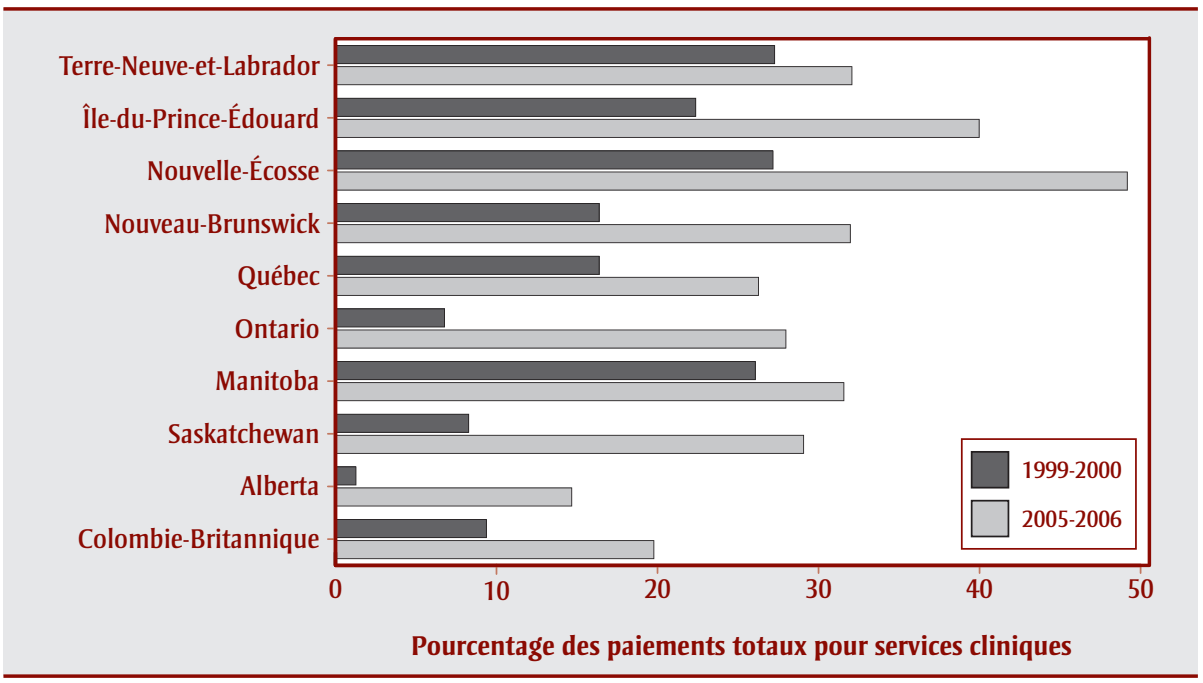

Source : Institut canadien d'information sur la santé. ${ }^{12}$ 
problème qui peut engendrer des lacunes dans les observations. L'hétérogénéité des systèmes de codage peut nuire à la consignation uniforme de certains types d'interventions, par exemple la pose d'un plâtre ou l'immobilisation d'un membre, qui servent à vérifier les cas de fractures ${ }^{23,24}$, ou encore les endoscopies ou colonoscopies, qui ont fait l'objet de recherches antérieures à l'échelle du Canada ${ }^{11}$.

\section{Points forts et limites de l'étude}

Bien que notre étude soit la première à décrire les caractéristiques des BDSM de plusieurs des provinces et territoires, elle n'est pas sans comporter certaines limites. Notre étude reposait sur un échantillon choisi par nos soins; il se pourrait que les informateurs clés n'aient pas eu toute l'information nécessaire sur les caractéristiques des BDSM, selon la période considérée ou la zone géographique concernée à l'intérieur d'une province ou d'un territoire. Autre limite : la qualité des données associées aux différentes caractéristiques n'a pas été examinée ${ }^{25}$. Par exemple, l'étude ne s'est pas penchée sur l'exactitude ou le caractère complet des champs renfermant les codes désignant les lieux de prestation des services.

Notre étude a mis en lumière un riche éventail d'occasions d'explorer davantage l'utilité et la comparabilité des BDSM dans le contexte des initiatives pancanadiennes de recherche et de surveillance. Dans le passé, la recherche sur la possibilité de biais causé par la durée de la période d'observation et le caractère incomplet des données d'estimation de la prévalence et de l'incidence s'est souvent concentrée sur les registres du cancer et sur les données venant d'une seule province ou d'un seul territoire ${ }^{26,27}$. Les techniques de simulation informatisée et de modélisation statistique qui ont été proposées pour estimer ou corriger ces effets ${ }^{28,29}$ pourraient être élargies à plusieurs provinces ou territoires. On pourrait aussi se servir des études méthodologiques sur l'utilité d'autres bases de données administratives, par exemple les bases de données sur les médicaments délivrés sur ordonnance, lorsqu'il s'agit d'évaluer l'exhaustivité des BDSM relativement à la surveillance des maladies chroniques ${ }^{8}$, ce qui permettrait d'établir des comparaisons entre plusieurs provinces et territoires. Dans une étude récente sur les méthodes permettant de confirmer les cas de maladie chronique dans les bases de données administratives sur la santé, on a conclu à la nécessité d'évaluer la validité des codes de diagnostic d'une population à l'autre et au fil du temps, pour s'assurer que les algorithmes de détection des cas pouvaient être généralisés ${ }^{30}$. D’autres caractéristiques des BDSM exigent un examen, notamment les procédures d'accès aux données, les codes d'honoraires et les procédures de couplage des données. Par exemple, on a constaté des différences marquées dans les honoraires applicables à la colonoscopie et à l'endoscopie à partir des barèmes d'honoraires des provinces et des territoires ${ }^{11}$. Il pourrait également y avoir des différences dans les types d'interventions et de services pour lesquels les codes d'honoraires ont été établis. Bien qu'un nombre croissant d'études fassent un couplage entre les BDSM et d'autres sources de données administratives, cliniques et tirées d'enquêtes sur la population ${ }^{31-33}$, nous n'avons pas examiné dans quelle mesure la capacité d'effectuer de tels couplages pourrait varier d'une province et d'un territoire à l'autre.

En résumé, cette étude a mis en lumière des différences dans un certain nombre de caractéristiques des BDSM d'une province et d'un territoire à l'autre. Ces différences pourraient nuire à la comparabilité des activités de recherche et de surveillance à l'échelle du Canada. Des études qui exploreraient l'effet potentiel de ces différences seraient utiles aux chercheurs, aux épidémiologistes et aux décideurs du domaine de la santé au Canada.

\section{Remerciements}

Cette étude a été réalisée grâce au financement fourni par les Instituts de recherche en santé du Canada à Lisa M. Lix et à H. Quan, et grâce aux fonds octroyés par le Centennial Research Chair Program de l'Université de la Saskatchewan à Lisa M. Lix. Les auteurs remercient les nombreuses personnes qui ont participé à la recherche, notamment Michael A. Ruta (gouvernement du Nunavut), la D ${ }^{\text {re }}$ Gillian Bartlett (Université McGill), Deanna Rothwell
(Hôpital d'Ottawa, Institut de recherche de l'Hôpital d'Ottawa et Institut de recherche en services de santé), Nedeene R. L. Hudema (Saskatchewan Health Quality Council), Nadine MacLean (Health PEI), la D ${ }^{\text {re }}$ Carol McClure (PEI Department of Health and Wellness), le $\mathrm{D}^{\mathrm{r}}$ Khokan C. Sikdar (Newfoundland and Labrador Centre for Health Information), Robert Fisk, Kim Reimer et Jenny Sutherland (Population Health Surveillance and Epidemiology Population and Public Health, BC Ministry of Health), Mike Tribes (expert-conseil, Yukon) et Charles Burchill (Manitoba Centre for Health Policy, Université du Manitoba).

Les auteurs désirent également remercier les chercheurs de l'équipe chargée de la surveillance et des résultats en matière d'hypertension, nommément la $D^{\text {re }}$ Karen Tu, la D ${ }^{\text {re }}$ Brenda Hemmelgarn, le $D^{r}$ Norman Campbell, le $\mathrm{D}^{\mathrm{r}}$ Finlay McAlister, le $\mathrm{D}^{\mathrm{r}}$ Michael Hill, la $\mathrm{D}^{\mathrm{re}}$ Nadia Khan, le $\mathrm{D}^{\mathrm{r}}$ Andreas Wielgosz, le $\mathrm{D}^{\mathrm{r}}$ Gary Teare, Mark Smith, Larry Svenson, le Dr Oliver Baclic, la $D^{\text {re }}$ Gillian Bartlett, la $D^{\text {re }}$ Sulan Dai, Jay Onysko et la $D^{\text {re }}$ Helen Johansen.

La présente étude repose en partie sur des renseignements fournis par le ministère de la Santé de la Saskatchewan. L'interprétation et les conclusions formulées par les auteurs ne représentent pas nécessairement la position du gouvernement de la Saskatchewan ou du ministère de la Santé de la Saskatchewan.

\section{Références}

1. Clottey C, Mo F, LeBrun B, Mickelson P, Niles J, Robbins G. La mise sur pied du Système national de surveillance du diabète (SNSD) au Canada. Maladies chroniques au Canada 2001;22:75-7.

2. Khan L, Mincemoyer S, Gabbay RA. Diabetes registries: where we are and where are we headed? Diabetes Technol Ther. 2009;11:255-62.

3. Dai S, Robitaille C, Bancej C, Loukine L, Waters C, Baclic O. Note de synthèse Rapport du Système national de surveillance des maladies chroniques : L'hypertension au Canada, 2010. Maladies chroniques au Canada 2010;31:46-7. 
4. Bernatsky S, Joseph L, Pineau CA, Bélisle P, Boivin JF, Banerjee D et collab. Estimating the prevalence of polymyositis and dermatomyositis from administrative data: age, sex, and regional differences. Ann Rheum Dis. 2009;68:1192-6.

5. Bernstein CN, Wajda A, Svenson LW et al. The epidemiology of inflammatory bowel disease in Canada: a population-based study. Am J Gastroenterol. 2006;101:1559-68.

6. Kisely S, Lin E, Lesage A, MacKenzie A, Koehoorn M, Jackson M et collab. Use of administrative data for the surveillance of mental disorders in 5 provinces. Can J Psychiatry. 2009;54:571-5.

7. Campbell N, Onysko J; Canadian Hypertension Education Program; Outcomes Research Task Force. The Outcomes Research Task Force and the Canadian Hypertension Education Program. Can J Cardiol. 2006;22:556-8.

8. Alshammari AM, Hux JE. The impact of non-fee-for-service reimbursement on chronic disease surveillance using administrative data. Can J Public Health. 2009;100:472-4.

9. Campbell N, Chen G. Canadian efforts to prevent and control hypertension. Can J Cardiol. 2010;26(Suppl C):14C-17C.

10. Miller E, Blatman B, Einarson TR. A survey of population-based drug databases in Canada. CMAJ. 1996;154:1855-64.

11. Roth LS, Adams PC. Variation in physician reimbursement for endoscopy across Canada. Can J Gastroenterol. 2009;23:503-5.

12. Institut canadien d'information sur la santé. Base de données nationale sur les médecins, 2008-2009. Ottawa (Ont.) : Institut canadien d'information sur la santé; 2010.

13. Institut canadien d'information sur la santé. Les médecins au Canada : État des programmes relatifs aux autres modes de paiement, 1999-2000. Ottawa (Ont.) : Institut canadien d'information sur la santé; 2001.

14. Institut canadien d'information sur la santé. Les médecins au Canada : État des programmes relatifs aux autres modes de paiement, 2005-2006. Ottawa (Ont.): Institut canadien d'information sur la santé; 2008.
15. Institut canadien d'information sur la santé. Profil des médecins selon le mode de rémunération : regard sur trois provinces. Ottawa (Ont.) : Institut canadien d'information sur la santé; 2010.

16. Downey W, Beck C, McNutt M, Stang M, Osei W, Nichol J. Health databases in Saskatchewan. Dans : Strom BL (dir.). Pharmacoepidemiology, $3^{\text {e }}$ éd. New York: Wiley; 2000;325-45.

17. Statistique Canada. $\mathrm{Au}$ sujet de l'Initiative sur les données longitudinales administratives et sur la santé. Série de documents de travail de la recherche sur la santé [Internet]. Ottawa (Ont.) : Statistique Canada; 2011 [consultation le 28 nov. 2011]. Consultable en ligne à la page : http:// www.statcan.gc.ca/pub/82-622-x/2011008 /intro-fra.htm

18. Statistique Canada. Comparabilité de la CIM-10 et de la CIM-9 pour les statistiques de la mortalité au Canada. 84-548-XIF [Internet]. Ottawa (Ont.) : Statistique Canada; 2005 [consultation le 28 nov. 2011]. Consultable en ligne à la page : http:// www.statcan.gc.ca/pub/ $84-548$ - x /84-548-x2005001-fra.htm

19. Parkin DM, Bray F. Evaluation of data quality in the cancer registry: principles and methods Part II. Completeness. Eur J Cancer. 2009;45:756-64.

20. Cox E, Martin BC, Van Staa T, Garbe E, Siebert U, Johnson ML. Good research practices for comparative effectiveness research: approaches to mitigate bias and confounding in the design of nonrandomized studies of treatment effects using secondary data sources: The International Society for Pharmacoeconomics and Outcomes Research Good Research Practices for Retrospective Database Analysis Task Force Report-Part II. Value in Health. 2009;12:1053-61.

21. Mullins IM, Siadaty MS, Lyman J, Scully K, Garrett CT, Miller WG et collab. Data mining and clinical data repositories: insights from a 667,000 patient data set. Comput Biol Med. 2006;36:1351-77.

22. Katz JN, Barrett J, Liang MH, Bacon AM, Kaplan H, Kieval RI et collab. Sensitivity and positive predictive value of Medicare Part B physician claims for rheumatologic diagnoses and procedures. Arthritis Rheum. 1997;40:1594-1600.
23. Leslie WD, Tsang JF, Lix LM. Validation of ten-year fracture risk prediction: a clinical cohort study from the Manitoba Bone Density Program. Bone 2008;43:667-71.

24. Jean S, Candas B, Belzile E, Morin S, Bessette L, Dodin S et collab. Algorithms can be used to identify fragility fracture cases in physician-claims databases. Osteoporos Int. 2012;23:483-501.

25. Karr AF, Sanil AP, Banks DL. Data quality: a statistical perspective. Stat Methodol. 2006;3:137-73.

26. McClish D, Penberthy L. Using multivariate capture-recapture techniques and statewide hospital discharge data to assess the validity of a cancer registry for epidemiologic use. Health Serv Outcomes Res Methodol. 2004;5:141-52.

27. Capocaccia R, de Angelis R. Estimating the completeness of prevalence based on cancer registry data. Stat Med. 1997;16:425-40.

28. Silcocks PB, Robinson D. Simulation modelling to validate the flow method for estimating completeness of case ascertainment by cancer registries. J Public Health. 2007;29:455-62.

29. Bernatsky S, Joseph L, Belisle P, Boivin JF, Rajan R, Moore A et collab. Bayesian modelling of imperfect ascertainment methods in cancer studies. Stat Med. 2005;24:2365-79.

30. Manuel DG, Rosella LC, Stukel TA. Importance of accurately identifying disease in studies using electronic health records. BMJ. 2010;341:c4226.

31. Lix LM, Yogendran MS, Shaw SY, Burchill C, Metge C, Bond R. Sources de données des populations utilisées en surveillance des maladies chroniques. Maladies chroniques au Canada 2008;29:34-42.

32. Muhajarine N, Mustard C, Roos LL, Young TK, Gelskey DE. Comparison of survey and physician claims data for detecting hypertension. J Clin Epidemiol. 1997;50:711-8.

33. Roos LL, Brownell M, Lix L, Roos NP, Walld R, MacWilliam L. From health research to social research: privacy, methods, approaches. Soc Sci Med. 2008;66:117-29. 